\title{
EDITORIAL
}

\section{Quo vadis Spectroscopy?}

\author{
Victor G. Mihucz (iD $\bigotimes$
}

Associate Professor \& Associate Editor of Brazilian Journal of Analytical Chemistry (BrJAC) Institute of Chemistry, ELTE - Eötvös Loránd University, Budapest, Hungary

Since the early days of modern chemistry, analytical chemistry always tried to offer solutions to reallife problems. This is how, in the midst of the Industrial Revolution, Margueritte developed the titrimetric determination of iron using the chameleon solution (potassium permanganate). In 1860, for the first time, Bunsen and Kirchhoff used element-specific light emission and absorption of flame-evaporated alkali metal salts for qualitative analysis. Their discovery was groundbreaking because the analytical sensitivity and detection capability of the proposed method was several orders of magnitude lower than that of the contemporary classical analytical ones. Application of this method, leading to the establishment of spectroscopic techniques, also allowed the discovery of about ten chemical elements. Another timeless merit of spectroscopy is the ability to determine the elemental composition of a star in a distant galaxy. In the second half of the $20^{\text {th }}$ century, an era of rapid development of instrumental analysis, atomic spectroscopy also brought several revolutionary results. One such breakthrough was the reduction of the sample volume required for analysis down to microliters with the introduction of graphite furnace atomic absorption spectrometry (GFAAS). The detection limit of GFAAS also decreased by several orders of magnitude. Another important advance was the combination of inductively coupled plasma as a high-temperature ion source with a mass spectrometer (ICP-MS). In the late 1980s, the hyphenation of atomic spectrometric devices to chromatographs, aiming at elemental speciation, also emerged. In Central and Eastern Europe, spectroscopic research was driven by the embargo of the Coordinating Committee for Multilateral Export Controls established by the Western Bloc during the Cold War. In this emerging era, scientific research and applications went hand in hand. Therefore, the science education program and fundamental research were very strong in those countries, contributing considerably to the development of spectrochemistry in Hungary. By the end of the $20^{\text {th }}$ century, development of new equipment and procedures was increasingly carried out by instrument manufacturers, and fundamental research at universities and institutes was relegated to second place. Analytical chemistry has experienced a considerable shift from determination of inorganic compounds and small organic molecules towards that of large (bio)molecules. Expansion of the application of analytical chemistry to forensic, environmental and biochemical questions has been observed and the role of atomic spectrometric techniques seemed to fade away. However, sheer use of these high-performance instruments is not always appropriate or cost-effective; the reliability of the results and elimination of interference must be thoroughly explored. However, when publishing such results, there is a risk that our communications will be rejected due to lack of novelty. Nevertheless, carrying out fundamental research cannot be avoided, as it is not possible to offer appropriate decisions to stakeholders based on questionable results. Presently, GFAAS and ICP-MS allow reliable quantitative determination of virtually any element in any sample. Recently, microwave plasma using nitrogen isolated from the air was launched on the market. This device requires significantly lower operating costs compared to the conventional ICP and reliable simultaneous multi-element analysis has also become possible. Another promising direction is single-particle ICP-MS applied for the characterization of inorganic nanoparticles. Most of the articles of

Cite: Mihucz, V. G. Quo vadis Spectroscopy? Braz. J. Anal. Chem., 2021, 8 (33), pp 1-2. doi: http://dx.doi.org/10.30744/ brjac.2179-3425.editorial.vgmihucz.N33 
the current issue of BrJAC also demonstrate that atomic spectrometry has become indispensable in many areas of our life. Enjoy reading the current issue!

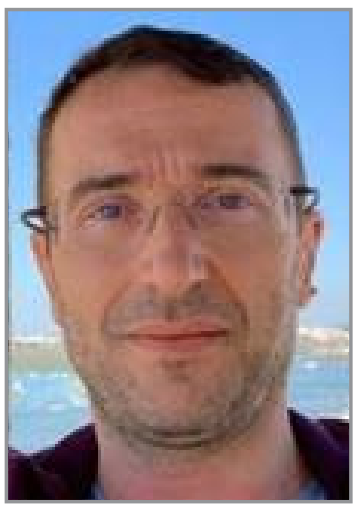

Victor Gábor Mihucz is currently Associate Professor at the Faculty of Science, Eötvös Loránd University, Budapest, Hungary. He works mainly in the field of Atomic Spectrometry, High-Performance Liquid Chromatography-Mass Spectrometry and Elemental Speciation, with emphasis on Environmental Analytical Chemistry. He is Editorial Board member for the Microchemical Journal and Applied Spectroscopy Reviews and was elected in 2018 as secretary of the Scientific Committee for Analytical and Environmental Chemistry of the Hungarian Academy of Sciences and as a member of the Steering Committee of the Hungarian Chemical Society (HCS) in 2019; he has been president of the Spectrochemical Association of HCS since 2019.

1 scopus 